East Tennessee State University

Digital Commons @ East Tennessee State University

\title{
Tracheobronchomalacia: An Unreported Pulmonary Complication of Acute Pancreatitis
}

\author{
Alexander Hwang \\ East Tennessee State University \\ Mahmoud El Iskandarani MD \\ East Tennessee State University - Department of Internal Medicine \\ Bara El Kurdi MD \\ East Tennessee State University - Department of Internal Medicine \\ Ibrahim Haddad MD \\ East Tennessee State University - Department of Internal Medicine \\ Sumbal Babar MD \\ East Tennessee State University - Department of Internal Medicine
}

Follow this and additional works at: https://dc.etsu.edu/asrf

Hwang, Alexander; El Iskandarani, Mahmoud MD; El Kurdi, Bara MD; Haddad, Ibrahim MD; and Babar, Sumbal MD, "Tracheobronchomalacia: An Unreported Pulmonary Complication of Acute Pancreatitis" (2020). Appalachian Student Research Forum. 10.

https://dc.etsu.edu/asrf/2020/presentations/10

This Oral Competitive is brought to you for free and open access by the Events at Digital Commons @ East Tennessee State University. It has been accepted for inclusion in Appalachian Student Research Forum by an authorized administrator of Digital Commons @ East Tennessee State University. For more information, please contactdigilib@etsu.edu. 


\section{Tracheobronchomalacia: An Unreported}

QUILLEN

COLLEGE of MEDICINE

COLLEGE of MEDICINE
Mahmoud El Iskandarani MD, Alexander Hwang BS, Ibrahim Haddad MD, Sumbal Babar MD, Bara El Kurdi MD

\section{Abstract}

Acute Pancreatitis (AP): a common disease of the pancreas with systemic complications, including well-defined pulmonary complications.

TBM: Results in an under-recognized diagnosis of airway collapse, which should be suspected in patients with Severe Acute Pancreatitis (SAP) who develop acute respiratory distress in whom no specific etiology has been determined.

While Severe Acute Pancreatitis (SAP) and its complications have been well-

documented, we believe this to be the first case report of

Tracheobronchomalacia (TBM) as a

respiratory complication of AP.

\section{Case Presentation}

- Patient Demographics: 54-yearold white male

- Medical history: diabetes mellitus with peripheral neuropathy and nephropathy as well as peripheral vascular disease and

- No history of pulmonary disease.

- Initial presentation: admitted for elective right below-knee amputation for diabetic osteomyelitis.

- Consulted: Internal medicine evaluated the patient for NAP of undetermined etiology complicating his post-operative course.

- Intervention: Treated per protocol with hydration, meropenem, and pain management.

- Complication(s): Within one week of NAP onset, the patient developed rapid respiratory distress.

\section{- Lab/lmaging Findings}

- ABG's were consistent with hypercapnic respiratory failure with hypoxemia and $\mathrm{PaO}_{2} / \mathrm{FiO}_{2}<$ 200.

- Chest radiography and ABG's did not support a diagnosis of ARDS.

- CT scan with IV contrast ruled out pulmonary embolism but showed a significant narrowing of the trachea with a drop from $4 \mathrm{~cm}$ in tracheal diameter on inspiration to $<1.7 \mathrm{~cm}$ on expiration when compared to a previous CT scan done three weeks earlier for a pulmonary nodule follow up. (Figure 1)

- Course: The patient's respiratory status continued to deteriorate requiring mechanical intubation with weaning trials proving to be futile. The patient eventually developed fungemia and expired after his family opted for palliative extubation.

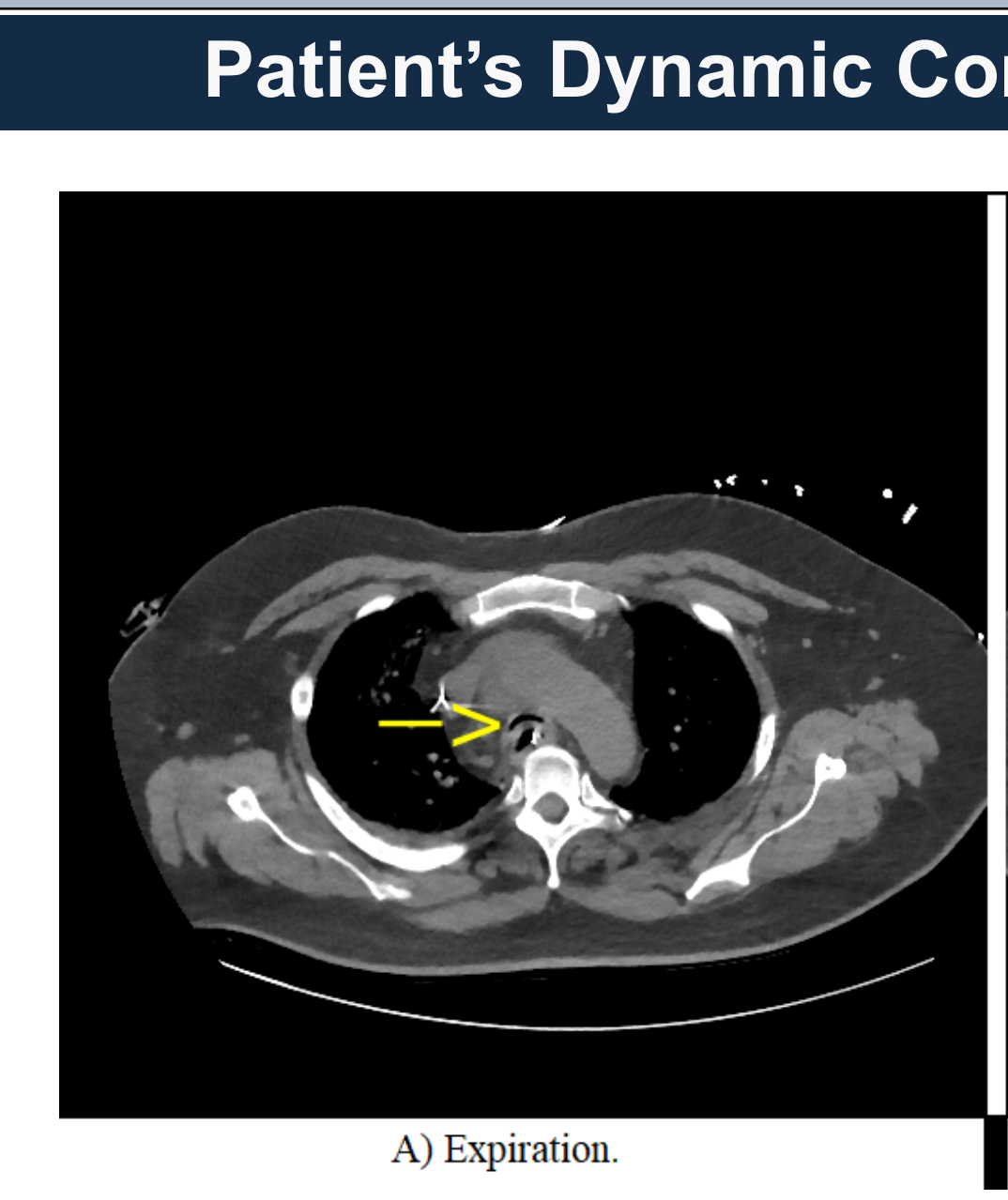

\section{Computed Tomography}

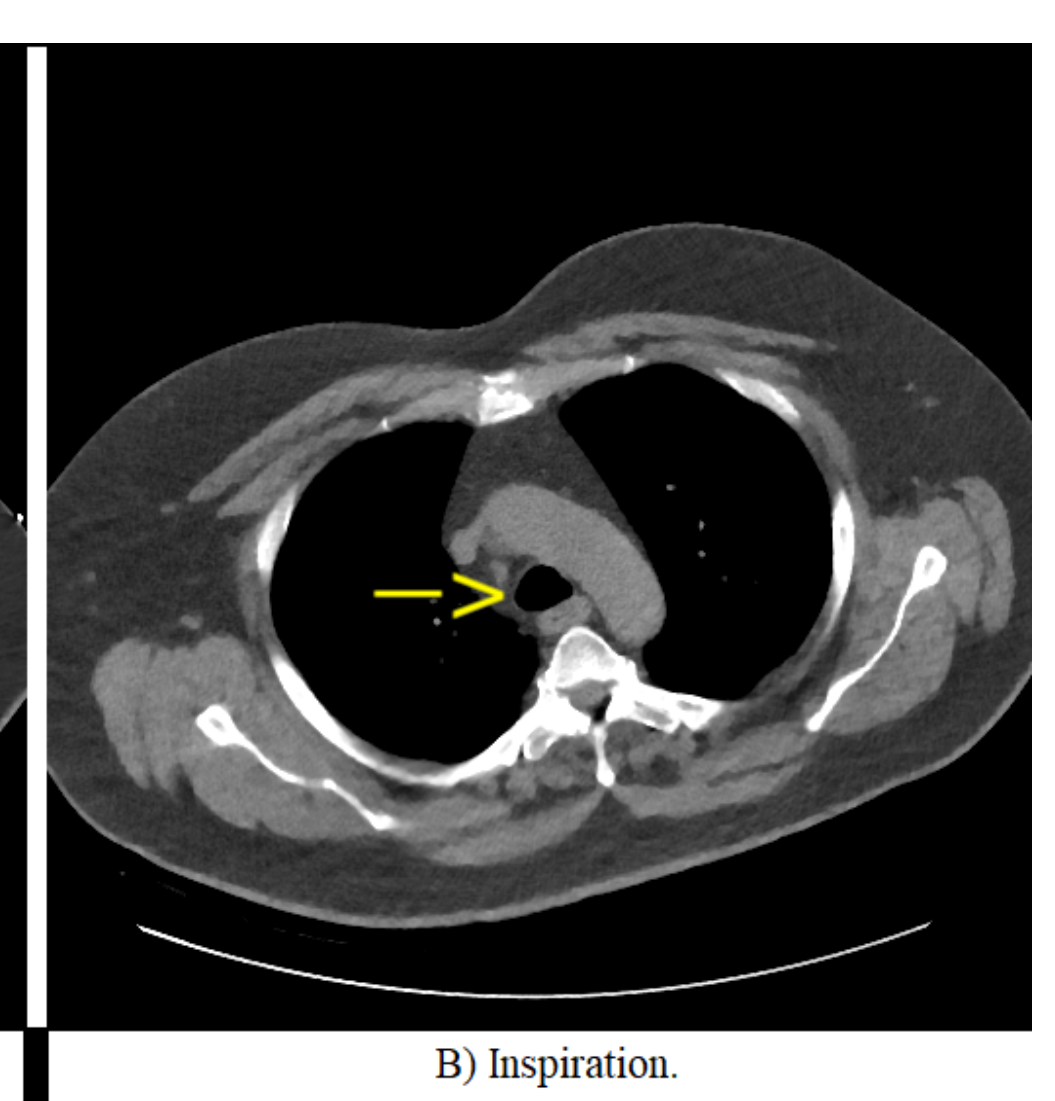

Figure 1: CT scan of the chest with IV contrast within one week of SAP onset Left: axial view during expiration

Right: axial view during inspiration

\section{Discussion}

Acute pancreatitis and severe pancreatitis differ in that severe pancreatitis is associated with organ failure, which can further be classified as moderate or severe, transient (resolves within 48 hours) or persistent (lasts more than 48 hours). If organ failure is persistent, rates of mortality are reported to reach $42 \%$. As described in the literature, the spectrum of pulmonary sequelae of severe acute pancreatitis ranges from a subclinical reduction in arterial oxygen tension to ARDS. The latter is often coupled with a poor prognosis, especially if ventilatory support is required.

TBM, a rare disease, is defined as a weakness of the trachea that is frequently due to either impaired cartilage integrity or reduction and/or atrophy of the longitudinal elastic fibers of the pars membranacea. A diagnosis of TBM can be made upon the finding of a dynamic airway collapse by dynamic flexible bronchoscopy (DFB), dynamic airway computed tomography (DACT), and/or pulmonary function testing. Furthermore, TBM is classified as either congenital or acquired. The congenital form is typically self-limiting by the age of two. The acquired form is secondary to recurrent infections, chronic inflammation from obstructive lung diseases, relapsing polychondritis, endobronchial intubation, tracheostomy, mechanical ventilation, lung transplant, and airway compression.

Our patient progressed from necrotizing acute pancreatitis to severe acute pancreatitis upon the onset of respiratory failure. After chest radiography and ABG's could not rule in ARDS, a CT scan with IV contrast of the chest was ordered to rule out a pulmonary embolism and instead revealed a dynamic instability of the main airways consistent with TBM. A comparison of the patient's prior CT scan of the chest revealed this was a new manifestation.

In our review of the literature, we believe this is the first reported case of TBM secondary to severe acute pancreatitis.

\section{Conclusion}

After an extensive review of the literature, we believe this to be the first report to describe the occurrence of TBM as a complication of SAP. Although it is unclear to the authors the impact of that diagnosis of this patient's outcome, TBM might have contributed to the failure of weaning trials off ventilation. In a patient with respiratory failure following severe acute pancreatitis, managing clinicians should be suspicious of a new-onset of TBM.

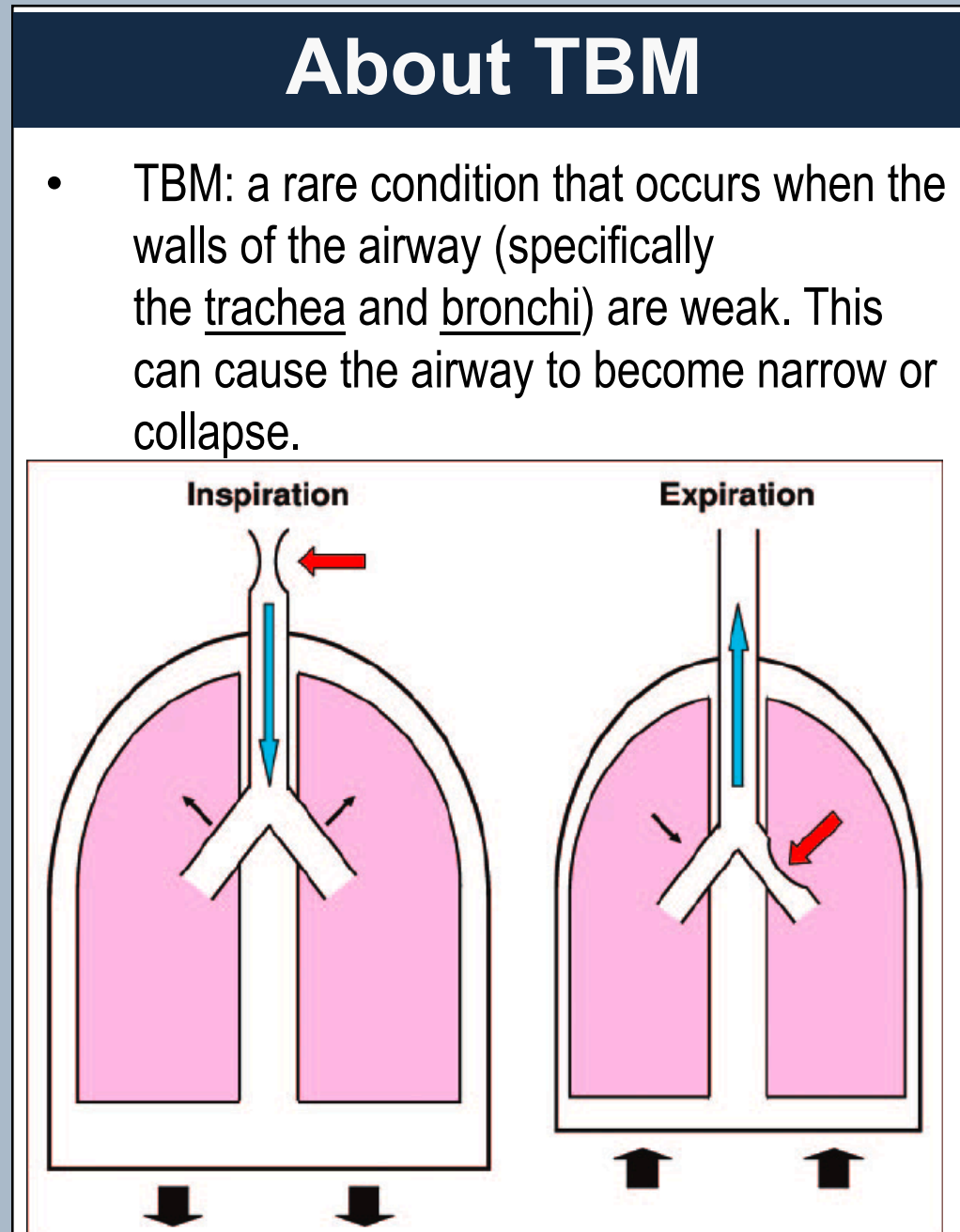

Acquired TBM is a rare occurrence. A variety of local and systemic diseases (such as recurrent infections, chronic inflammation from obstructive lung diseases, relapsing polychondritis, endobronchial intubation, tracheostomy, mechanical ventilation, lung transplant, and airway compression) have been linked to its development.

\section{References}

Maruti Govindappa Raghu, Jai Dev Wig Rakesh Kochhar, et al.: Lung complications in acute pancreatitis. JOP J. Pancreas. 8:177-185

Peter A. Banks, Thomas L Bollen, Christos Dervenis, et al.: Classification of acute pancreatitis-2012: revision of the Atlanta classification and definitions by international consensus. Gut. 62:102-111.

Kelly A. Carden MD, Philip M. Boiselle MD FCCP, David A. Waltz MD, et

al. : Tracheomalacia and

Tracheobronchomalacia in Children and Adults: An In-depth Review. CHEST. 127:984-1005

Adnan Majid, Kumar Gaurav, Jully M. Sanchez, et al.: Evaluation of tracheobronchomalacia by dynamic flexible bronchoscopy. A pilot study. Ann. Am. Thorac. Soc. 11:951-955

Karen S. Lee MD, Maryellen R.M. Sun MD, Armin Ernst MD FCCP, et al: Comparison of Dynamic Expiratory CT With Bronchoscopy for Diagnosing Airway Malacia: A Pilot Evaluation. Chest. 131:758-764

Tracheobronchomalacia. National Center for Advancing Translational Sciences. https://rarediseases.info.nih.gov/diseases 17791/tracheobronchomalacia.

Vicencio, Alfin \& Parikh, Sanjay. (2006). Laryngomalacia and Tracheomalacia: Common Dynamic Airway Lesions. Pediatrics in review / American Academy of Pediatrics. 27. e33-5; discussion e35. 10.1542/pir.27-4-e33.

\section{Acknowledgements}

Special thanks to the Department of Interna Medicine Department, the Quillen College of Medicine, East Tennessee State University, Dr. Jennifer Treece, and Dr. Bara El Kurdi for their collective support throughout this report. 